\title{
THE ESTIMATION OF NEIGHBOURHOOD PARAMETERS FOR PLANT POPULATIONS
}

\author{
T. J. CRAWFORD \\ Department of Biology, University of York, Heslington, York YO150D, U.K.
}

Received 20.ix.83

\section{SUMMARY}

\begin{abstract}
Sewall Wright's neighbourhood model indicates that the area containing a panmictic unit within a continuous and uniform array of organisms can be estimated by $4 \pi \sigma^{2}$ where $\sigma^{2}$ is the parent-offspring dispersal variance measured around a zero mean and relative to a single reference axis passing through the population. The method has proved popular in studies on higher plants but the literature is confused as to how the two components of dispersal variance, for pollen and seeds, should be combined. It is argued that $\sigma^{2}=\frac{1}{2} \sigma_{p}^{2}+\sigma_{s}^{2}$ where $\sigma_{p}^{2}$ and $\sigma_{s}^{2}$ are pollen and seed dispersal variances already corrected to give axial variances. The consequences of using different methods are compared and some puzzling aspects of the previous literature are discussed.
\end{abstract}

\section{INTRODUCTION}

Wright $(1943,1946)$ considered the effects of restricted gene dispersal upon the genetic properties of a population distributed continuously and uniformly in space. The important parameter was the genetically effective number of individuals $\left(N_{e}\right)$ in a "neighbourhood", defined as an area from which the parents of central individuals may be treated as if drawn at random. He concluded that if $N_{e}$ was as small as 20 there was considerable scope for random local genetic differentiation within the population; with $N_{e}$ of the order of 200 a moderate amount of differentiation would be expected, but with $N_{e}$ as large as 1000 there would, in effect, be universal panmixia. These figures apply to an area continuum; in a linear population marked differentiation is expected with $N_{e}$ as large as $10^{4}$. Computer simulations (Rohlf and Schnell, 1971) give qualitative support to these conclusions.

The spatial dimensions of a neighbourhood are a function of the variance of the parent-offspring dispersal distribution. In principle, at least, this variance is easily estimated for plants because usually there are only two components of dispersal, through pollen and seed. Nevertheless, there is confusion in the literature as to how these two components should be combined to yield the parent-offspring dispersal variance. At least three methods have been proposed without any assessment of their relative merits. In view of the current popularity of studies on neighbourhood structure in plants, it is worth considering which, if any, of these three methods is correct. This paper will show that, for both statistical and biological reasons, all three methods are incorrect.

\section{Wright'S NeIghbourhood MODEL}

Attention will be concentrated on the areal model; results for the linear model will be compared where relevant. Individuals are considered to be 
distributed uniformly throughout the area and, for simplicity, are assumed to be hermaphrodite and mating at random, including self fertilisation at its appropriate frequency $(1 / N)$. If parent-offspring dispersal distances follow a zero mean circular normal distribution with variance $\sigma^{2}$ along any axis, the neighbourhood is a circle of radius $2 \sigma$ and has an area $A=4 \pi \sigma^{2}$ (Wright, 1946, 1969). The number of individuals within the neighbourhood is $N=A d$ where $d$ is the density of breeding individuals. If the effective number of individuals per unit area $\left(d_{e}\right)$ is estimated, the effective neighbourhood number is $N_{e}=A d_{e}$.

For a linear population where parent-offspring dispersals follow a univariate zero mean normal distribution with variance $\sigma^{2}$, the neighbourhood length is $L=2 \pi^{1 / 2} \sigma$. If $d_{e}=$ the effective number of individuals per unit length, again $N_{e}=L d_{e}$.

The model makes a number of assumptions, in particular that dispersal distances are normally distributed, that the distribution has a zero mean (i.e., no net displacement of the population over time) and that the components of dispersal as measured accurately refiect actual gene dispersal. These assumptions are considered by Crawford (1984); for present purposes they are taken to hold.

\section{The Estimation of $\sigma^{2}$}

The required variance is that of dispersals measured relative to a single axis passing through the population and its estimation is discussed by Kerster (1964). Wright (1978) refers to this variance as the "one-way variance" but it has more generally been called the axial variance.

Three methods have been used to estimate $\sigma_{\text {axial. }}^{2}$.

(i) Dispersal distances may be measured relative to an arbitrary pair of rectangular axes, $x$ and $y$. If $n$ observations are made, then relative to the $x$-axis

$$
\sigma_{x}^{2}=\Sigma(x-\bar{x})^{2} / n=\Sigma x^{2} / n
$$

because of the zero mean assumption. Similarly, $\sigma_{y}^{2}=\Sigma y^{2} / n$. These axial variances have equal expectations and their average is taken as the estimate:

$$
\sigma_{\text {axial }}^{2}=\left(\Sigma x^{2}+\Sigma y^{2}\right) / 2 n \text {. }
$$

(ii) It is usually more practicable to measure absolute radial dispersal $h$ without reference to specific axes. The zero mean assumption requires that absolute dispersals occur at angles $\theta$ to an arbitrary axis so that $\theta$ takes random values between $0^{\circ}$ and $360^{\circ}$.

$$
\begin{aligned}
\sigma_{\text {absolute }}^{2}=\Sigma h^{2} / n & =\Sigma\left(x^{2}+y^{2}\right) / n \text { by Pythagoras } \\
& =\left(\Sigma \mathrm{x}^{2}+\Sigma \mathrm{y}^{2}\right) / n \\
& =2 \sigma_{\text {axial }}^{2},
\end{aligned}
$$

i.e., the required variance is one half of the variance of absolute dispersal distances.

(iii) Wind dispersal of seed is sometimes estimated along a single axis by placing plants on the windward side of an expanse of cheesecloth onto which the seeds adhere. Measured dispersal is in a positive direction only 
and the zero mean assumption requires that each observation is balanced by one of negative sign, but equal absolute value, along the same axis (e.g., Levin and Kerster, 1969a). Then

$$
\sigma_{\text {axial }}^{2}=2 \Sigma x^{2} / 2 n=\Sigma x^{2} / n
$$

The variance around the observed mean dispersal, as used in Levin and Kerster (1968), is inappropriate.

Estimates of pollen and seed dispersal variances, $\sigma_{p}^{2}$ and $\sigma_{s}^{2}$, must be combined to yield an estimate of the axial parent-offspring dispersal variance for substitution in $A=4 \pi \sigma_{\text {axial. }}^{2}$. Three different methods have been proposed. For ease of comparison, it is assumed that absolute variances have already been corrected to axial variances where required. Levin and Kerster (1968) summed the pollen and seed variances so that

$$
\sigma_{\text {axial }}^{2}=\sigma_{p}^{2}+\sigma_{s}^{2} \text {. }
$$

In later papers (Levin and Kerster, 1969a, b, 1971, 1975) they averaged the pollen and seed variances:

$$
\sigma_{\text {axial }}^{2}=\frac{1}{2}\left(\sigma_{p}^{2}+\sigma_{s}^{2}\right) \text {. }
$$

A further refinement (Levin and Kerster, 1974; Levin, 1978, 1979) is to halve the pollen variance, after averaging, to allow for pollen being haploid and seed diploid:

$$
\sigma_{\text {axial }}^{2}=\frac{1}{2}\left(\frac{1}{2} \sigma_{p}^{2}+\sigma_{s}^{2}\right) .
$$

Expression (ii) has proved most popular in practice (e.g., Richards and Ibrahim, 1978; Schaal and Levin, 1978; Schmitt, 1980).

None of these methods is related to the nature of paternal and maternal gene dispersal in plants: paternal dispersal is through pollen and seed, whereas maternal dispersal is through seed only. Male gamete dispersal variance is $\sigma_{p}^{2}$ and female gamete dispersal variance is 0 , at least in higher plants. The average gamete dispersal variance, is, therefore, $\frac{1}{2}\left(\sigma_{p}^{2}+0\right)=\frac{1}{2} \sigma_{p}^{2}$. Seed dispersal is a post-fertilisation event and should be regarded as progeny dispersal so that the total parent-offspring dispersal variance is

$$
\sigma_{\text {axial }}^{2}=\frac{1}{2} \sigma_{p}^{2}+\sigma_{s}^{2} \text {. }
$$

The validity of expression (iv) is confirmed by a statistical examination of the situation (see fig. 1). For a given case absolute pollen and seed dispersals, $p_{h}$ and $s_{h}$, are shown relative to $x$ - and $y$-axes. The female parent-offspring dispersal is $s_{h}$ but the required male parent-offspring dispersal is that measured directly $\left(m_{h}\right)$. Taking signed directions into account

$$
\begin{gathered}
m_{x}=s_{x}+p_{x} \text { and } m_{y}=s_{y}+p_{y} . \\
\therefore \quad m_{h}=\left[\left(s_{x}+p_{x}\right)^{2}+\left(s_{y}+p_{y}\right)^{2}\right]^{1 / 2} .
\end{gathered}
$$

If female parent- and direct male parent-offspring dispersal variances are $\sigma_{q}^{2}$ and $\sigma_{\delta}^{2}$, the combined parent-offspring variance is

$$
\sigma^{2}=\frac{1}{2}\left(\sigma_{\delta}^{2}+\sigma_{\wp}^{2}\right)
$$

as each offspring has one male and one female parent. $\sigma_{q}^{2}=\sigma_{s}^{2}$ but we have yet to find $\sigma_{\delta}^{2}$. 


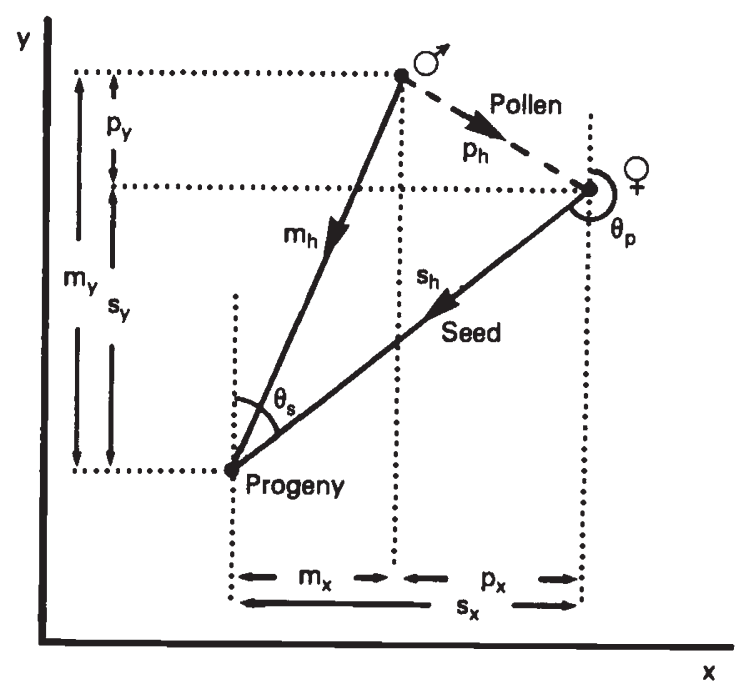

FIG. 1. Pollen, seed and direct male dispersals measured absolutely and relative to a pair of arbitrary $x$ - and $y$ - axes.

Working in terms of axial variances only (absolute variances yield the same answer):

Similarly,

$$
\begin{aligned}
\sigma_{m, x}^{2} & =\Sigma m_{x}^{2} / n \\
& =\Sigma\left(s_{x}+p_{x}\right)^{2} / n \\
& =\left(\Sigma s_{x}^{2}+\Sigma p_{x}^{2}\right) / n+2 \Sigma s_{x} p_{x} / n \\
& =\sigma_{s, x}^{2}+\sigma_{p, x}^{2}+2 \operatorname{cov}\left(s_{x}, p_{x}\right) .
\end{aligned}
$$

Now

$$
\sigma_{m, y}^{2}=\sigma_{s, y}^{2}+\sigma_{p, y}^{2}+2 \operatorname{cov}\left(s_{y}, p_{y}\right) \text {. }
$$

$$
\begin{aligned}
\sigma_{\delta}^{2} & =\frac{1}{2}\left(\sigma_{m, x}^{2}+\sigma_{m, y}^{2}\right) \\
& =\frac{1}{2}\left(\sigma_{s, x}^{2}+\sigma_{s, y}^{2}\right)+\frac{1}{2}\left(\sigma_{p, x}^{2}+\sigma_{p, y}^{2}\right)+\operatorname{cov}\left(s_{x}, p_{x}\right)+\operatorname{cov}\left(s_{y}, p_{y}\right) \\
& =\sigma_{s}^{2}+\sigma_{p}^{2}+\text { covariances. }
\end{aligned}
$$

The covariances cannot be estimated because in practice a given seed dispersal cannot be connected to the specific pollen dispersal that lead to fertilisation of that ovum. In any case, the covariances have statistical expectations of zero and this is likely to hold in nature. Therefore,

$$
\begin{aligned}
\sigma_{\text {axial }}^{2} & =\frac{1}{2}\left(\sigma_{\delta}^{2}+\sigma_{\S}^{2}\right) \\
& =\frac{1}{2}\left(\sigma_{s}^{2}+\sigma_{p}^{2}+\sigma_{s}^{2}\right) \\
& =\frac{1}{2} \sigma_{p}^{2}+\sigma_{s}^{2} .
\end{aligned}
$$

\section{Computer Simulation}

Gene dispersal was simulated backwards from $n$ progeny, all at position $x=0, y=0$, to female and male parents. For a circular normal distribution 


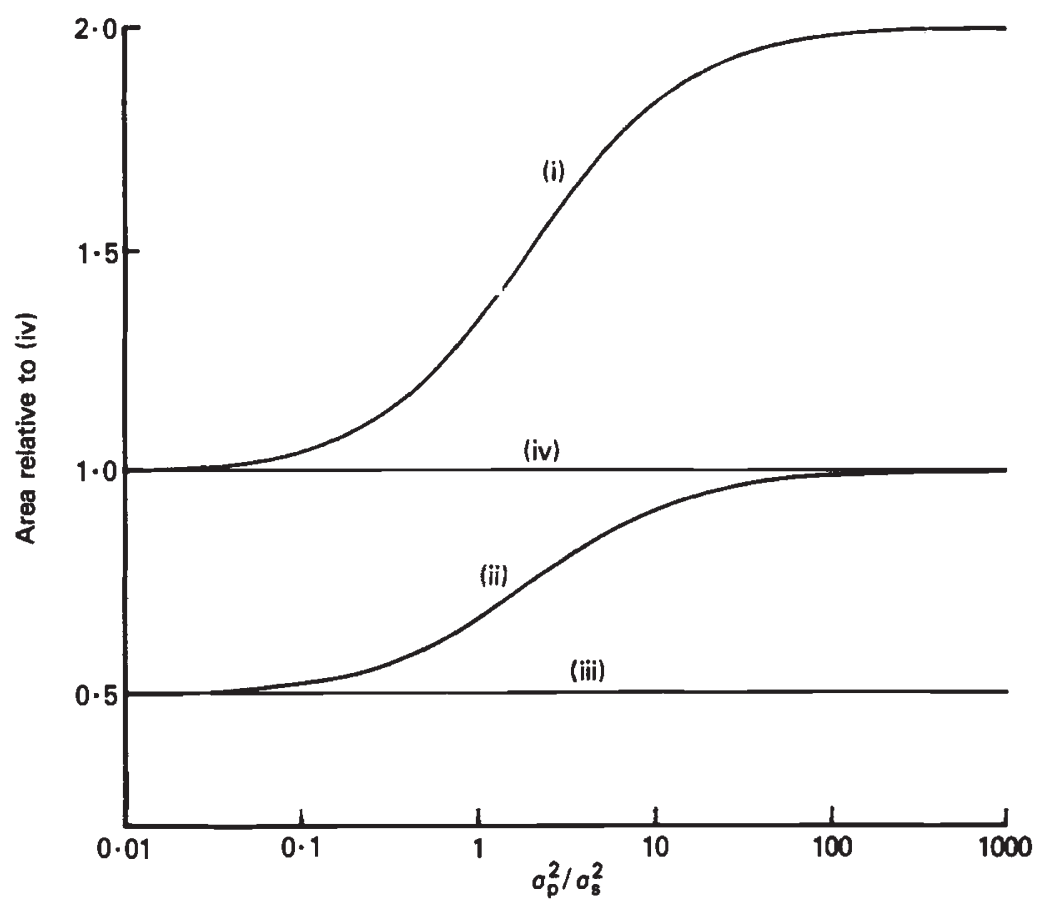

FIG. 2. Neighbourhood areas estimated by expressions (i) to (iv) as ratios to those estimated by expression (iv).

the proportion of random observations that lie within a circle of radius $r \sigma$ is $P_{r}=1-\exp \left(-r^{2} / 2\right)$. Seed dispersal at a random angle $\theta_{s} \in[0,2 \pi]$ radians to the $y$-axis was, therefore, simulated with length $s_{h}=$ $\left[-2 \log _{e}\left(1-P_{r}\right) \sigma_{s}^{2}\right]^{1 / 2}$, random $P_{r} \in[0,1]$ and $\sigma_{s}^{2}=$ expected axial seed dispersal variance. By repeating this process the positions of the $n$ female parents were fixed. Pollen dispersals, length $p_{h}$, were likewise simulated from each female parent to the corresponding male parent using random angles $\theta_{p}$ and $\sigma_{p}^{2}$, the expected axial pollen dispersal variance (see fig. 1). The male parent-offspring simulated dispersals are

$$
\begin{aligned}
& m_{x}=s \sin \theta_{s}+p \sin \theta_{p}, \\
& m_{y}=s \cos \theta_{s}+p \cos \theta_{p} \\
& m_{h}=\left[s_{h}^{2}+p_{h}^{2}+2 s_{h} p_{h} \cos \left(\theta_{s}-\theta_{p}\right)\right]^{1 / 2} .
\end{aligned}
$$

Simulations, size $n=1000$ progeny, were conducted for a range of values of $k=\sigma_{p}^{2} / \sigma_{s}^{2}$ and the observed axial and absolute variances were examined. In all cases the male parent-offspring dispersal variance agreed closely with $\sigma_{p}^{2}+\sigma_{s}^{2}$ as predicted. The male parent-offspring dispersals were combined with the female parent-offspring (i.e., seed) dispersals to yield a single parent-offspring dispersal distribution of $2 n$ observations. The variance of this distribution agreed with $\frac{1}{2} \sigma_{p}^{2}+\sigma_{s}^{2}$, irrespective of $k$, and provided further confirmation that pollen and seed variances should be combined according to expression (iv).

Using the notation $\sigma_{1}^{2}, \ldots, \sigma_{4}^{2}$ to refer to $\sigma^{2}$ as defined by expressions (i) to (iv) respectively, fig. 2 shows the areas of neighbourhoods estimated 
by the four methods, relative to $A=4 \pi \sigma_{4}^{2}=1$, as functions of $k=\sigma_{p}^{2} / \sigma_{s}^{2}$. $\sigma_{1}^{2} / \sigma_{4}^{2}=2(k+1) /(k+2)$ and expression (i) is adequate when $\sigma_{p}^{2} \ll \sigma_{s}^{2}$, otherwise it overestimates by a factor of up to 2 . $\sigma_{2}^{2} / \sigma_{4}^{2}=(k+1) /(k+2)$ and expression (ii) is reasonable when $\sigma_{p}^{2} \gg \sigma_{s}^{2}$, otherwise it leads to estimates of down to one-half the correct estimate. $\sigma_{3}^{2} / \sigma_{4}^{2}=\frac{1}{2}$, irrespective of $k$, so that expression (iii) always underestimates by a factor of 2 .

A second program simulated gene dispersal in a linear neighbourhood. Absolute dispersals are irrelevant; seed and pollen dispersals along a single axis were drawn at random from univariate normal distributions with variances $\sigma_{s}^{2}$ and $\sigma_{p}^{2}$ by the polar method (Knuth, 1981). The variance of the combined parent-offspring dispersal distribution again agreed with $\frac{1}{2} \sigma_{p}^{2}+\sigma_{s}^{2}$. As $L=2 \pi^{1 / 2} \sigma$, comparisons between estimates of $L$ using $\sigma_{1}^{2}, \ldots, \sigma_{4}^{2}$ are qualitatively the same as in the areal model but the differences between estimates are the square root of those shown in fig. 2. For example, $\sigma_{1}^{2} / \sigma_{4}^{2}=[2(k+1) /(k+2)]^{1 / 2}$ so that expression (i) overestimates, by a factor of up to $2^{1 / 2}$, except when $\sigma_{p}^{2} \ll \sigma_{s}^{2}$.

\section{The Proportion of Central Individuals' Parents Within the NEIGHBOURHOOD}

Following Wright (1946), it has often been stated that a neighbourhood of area $4 \pi \sigma^{2}$ will include within the panmictic circle of $2 \sigma$ radius 86.5 per zent of the parents of individuals at the neighbourhood's centre (Levin and Kerster, 1971, 1974; Levin, 1978, 1979; Schaal and Levin, 1978). This is only true, however, if the parent-offspring dispersal variances are the same for parents of both sexes as assumed by Wright. This will rarely be the case for higher plants as $\sigma_{q}^{2}=\sigma_{s}^{2}$ whereas $\sigma_{\delta}^{2}=\sigma_{p}^{2}+\sigma_{s}^{2}$. Thus $\sigma_{\delta}^{2}>\sigma_{q}^{2}$ unless $\sigma_{p}^{2}=0$ when $\sigma_{\delta}^{2}=\sigma_{q}^{2}$. If gametes alone are considered the male dispersal variance is $\sigma_{p}^{2}$ and that for female gametes is always zero.

Although Wright $(1946,1969)$ considers unequal dispersion of the sexes in the context of plants, the distinction between parent-offspring, gamete (pollen) and seed dispersal variances is not always clear. He takes the extreme case where all dispersal is via pollen (i.e., $\sigma_{s}^{2}=0$ ) and finds that $A=4 \pi \sigma_{p}^{2} / 2$ in agreement with expressions (ii) and (iv). Then a proportion $1-e^{-1}=0.63$ of the parents of central individuals are expected to lie in the neighbourhood. But the conclusion that neighbourhood area is half as great as when male and female gametes show the same dispersal variance as male gametes in the case in question cannot be correct for plants. As shown above, male and female gametes have the same dispersal variances only when $\sigma_{p}^{2}=0$ so that the equivalent model with pollen dispersal alone would involve no gene dispersal at all. Neighbourhood area is, in fact, twice as great when, in addition to pollen dispersal with the same variance, there is also seed dispersal with variance half as great as that of the pollen so that $\sigma_{\delta}^{2}=3 \sigma_{q}^{2}$ for parent-offspring dispersal.

The proportion of central individuals' parents that lie within the neighbourhood depends upon the ratio $\sigma_{p}^{2} / \sigma_{s}^{2}=k$. The proportion of female parents within the radius $2 \sigma$

$$
=1-e^{-r_{2}^{2} / 2}=1-e^{-(2+k)}
$$

as $r_{q}=2 \sigma / \sigma_{q}$. Similarly the proportion of male parents within the radius $2 \sigma$

$$
=1-e^{-r_{\delta}^{2} / 2}=1-e^{-(2+k) /(1+k)} \text {. }
$$




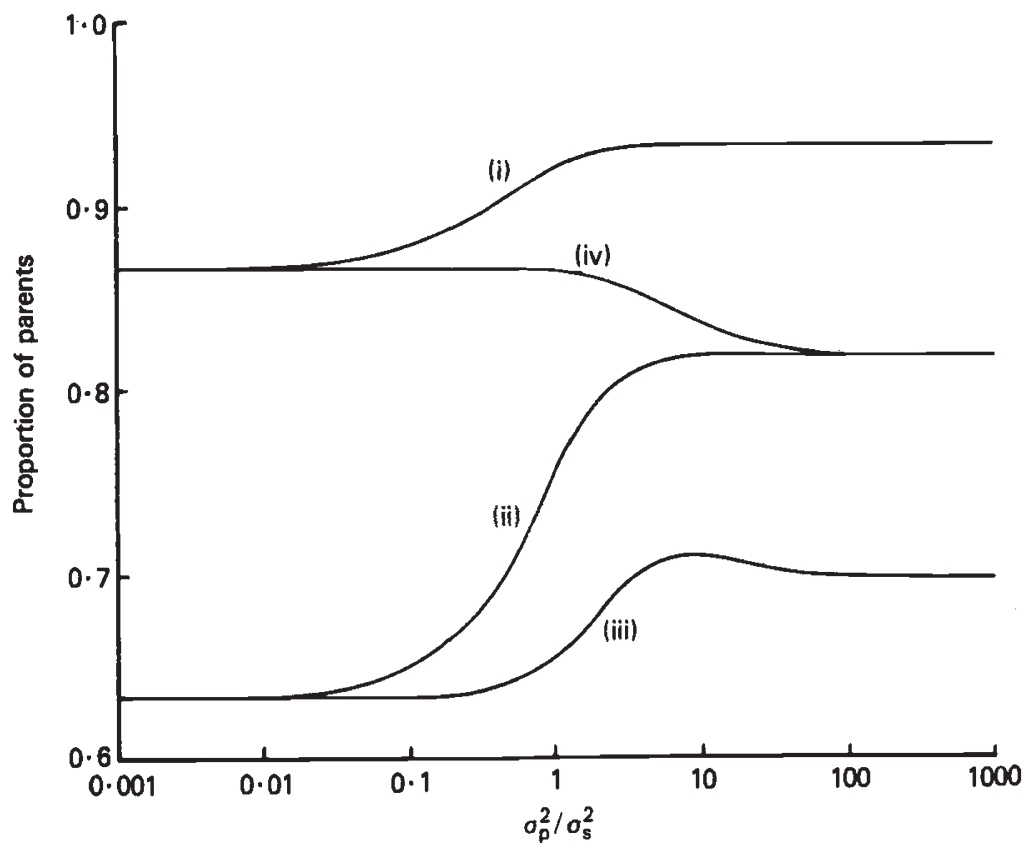

FIG. 3. Expected proportion of central individuals' parents lying within a neighbourhood area for $\sigma^{2}$ estimated by expressions (i) to (iv).

Therefore, as each central offspring has one parent of each sex, the proportion of their parents, female or male, lying within the neighbourhood is

$$
\frac{1}{2}\left[\left\{1-e^{-(2+k)}\right\}+\left\{1-e^{-(2+k) /(1+k)}\right\}\right] .
$$

The expected proportion of parents as a function of $k$ (or $\sigma_{\delta}^{2} / \sigma_{q}^{2}=k+1$ ) is shown in fig. 3. Approximately, when $k<1,0 \cdot 865$ of these parents lie within the neighbourhood; otherwise the proportion falls to a limit of 0.816 when $\sigma_{p}^{2} \gg \sigma_{s}^{2}$. This limit is the average of 1.0 for female parents and 0.632 for male parents, the figures appropriate for the case of pollen dispersal only. These predictions, based on $\sigma^{2}$ as defined in expression (iv) were confirmed in the computer simulations; for comparison, fig. 3 also shows the expected proportions of parents when $\sigma^{2}$ is calculated using expressions (i) to (iii).

For the linear model the equivalent proportions of central individuals' parents within the neighbourhood are less easily described. If $\sigma^{2}$ is estimated according to expression (iv) the proportion of parents is

$$
\frac{1}{2}\left[\left\{1-P\left(d_{q}\right)\right\}+\left\{1-P\left(d_{\delta}\right)\right\}\right]
$$

where $P\left(d_{q}\right)$ and $P\left(d_{\delta}\right)$ are the two-tailed probabilities of the standardised normal deviate exceeding

$$
\left[\frac{\pi}{2}(2+k)\right]^{1 / 2} \text { and }\left[\frac{\pi}{2}\left(\frac{2+k}{1+k}\right)\right]^{1 / 2}
$$

respectively. 92.4 per cent of parents lie within the neighbourhood when $k<1$. For $k>1$ the figure declines to a limit of 89.5 per cent. If expressions 
(i) to (iii) are used for $\sigma^{2}$ the differences are less than for the area model, although qualitatively similar.

Returning to the area model, it has been suggested that, as a matter of definition, 50 per cent of a neighbourhood's genes are replaced from outside each generation (Kerster and Levin, 1968; Levin and Kerster, 1968, 1969a). This follows from Dobzhansky and Wright (1943) where they concluded, incorrectly, that the neighbourhood radius was $2^{1 / 2} \sigma$ so that 36.8 per cent of central individuals' parents would lie outside the neighbourhood. As considerably more than 50 per cent of marginal individuals' parents would come from outside, they suggested about 50 per cent replacement of the neighbourhood population per generation by local immigrants probably differing little in gene frequencies. Wright (1946) drew attention to the error and showed that the correct radius was $2 \sigma$. The level of gene replacement is, therefore, likely to be less than that suggested by Dobzhansky and Wright and will depend on $\sigma_{p}^{2} / \sigma_{s}^{2}$. Indeed, in the case of Lithospermum caroliniense (Kerster and Levin, 1968) the assumption $\sigma_{s}^{2}=0$ was made; all female parents must lie inside the neighbourhood, so for 50 per cent gene replacement all male parents would have to lie outside the neighbourhood.

\section{Discussion}

The basic neighbourhood model discussed in this paper is an ideal situation rarely achieved in nature: the consequences for neighbourhood area estimation of the main assumptions failing to hold have been discussed by Crawford (1984). Nevertheless, some of the published neighbourhood areas for herbaceous plants are strikingly small, for example $4-26 \mathrm{~m}^{2}$ for Lithospermum caroliniense (Kerster and Levin, 1968) and $2 \cdot 8-6.3 \mathrm{~m}^{2}$ for Lupinus texensis (Schaal, 1980). Such small estimates suggest that different parts of extensive colonies will be separated by many neighbourhood diameters leading to high levels of isolation and promoting genetic differentiation. The effective number of individuals in neighbourhoods will determine the extent to which differentiation reflects responses to local selective forces or the consequences of random genetic drift. Estimated neighbourhood sizes were $2-7$ for $L$. caroliniense and $42-95$ for $L$. texensis; effective neighbourhood sizes may well be significantly smaller. Almost no information on effective number is available for plant populations, although Mackay (1980) found female fecundity variation between Papaver dubium individuals to be so great that $N_{e}$ would be about an order of magnitude lower than $N$ for this reason alone. It seems likely, therefore, that effective neighbourhood numbers may often be sufficiently small for random local genetic differentiation to occur in plant populations. In view of this it is important that neighbourhood parameters should be estimated accurately within the limitations of the methods involved.

Levin and Kerster (1968) estimated neighbourhood areas for three colonies of Phlox pilosa by application of expression (i). Taking their non-truncated figures for colony I, 136 pollinator flights were observed to have a mean of $1.42 \mathrm{~m}$ and a variance of $10.84 \mathrm{~m}^{2}$. Axial dispersal distance was measured in the laboratory for 395 seeds giving a mean of $1.12 \mathrm{~m}$ and a variance of $0.54 \mathrm{~m}^{2}$. Correcting for absolute pollen measurements $\sigma_{p}^{2}=$ $5.42 \mathrm{~m}^{2}$ and $\sigma_{s}^{2}=0.54 \mathrm{~m}^{2} . \sigma^{2}=\sigma_{\mathrm{p}}^{2}+\sigma_{\mathrm{s}}^{2}=5.96 \mathrm{~m}^{2}$ and $A=75 \mathrm{~m}^{2}$. The 
density of plants was $13 \cdot 1 \mathrm{~m}^{-2}$ so that $N=983$. As $\sigma_{p}^{2} / \sigma_{s}^{2} \simeq 10$ these figures will be about 1.8 times as large as if expression (iv) is employed (fig. 2). However, the standard error of the mean pollinator flight distance is $0.28 \mathrm{~m}$ suggesting that the variance is that around the observed mean, ignoring direction, so that the appropriate absolute variance, assuming a zero mean, is $[(135)(5 \cdot 42) / 136]+1 \cdot 42^{2}=12 \cdot 78 \mathrm{~m}^{2}$. Similarly, $\sigma_{s}^{2}$ appears to be estimated relative to the observed mean and measurements from the histogram (Levin and Kerster, fig. 3) suggest that axial $\sigma_{s}^{2}$ is about $2.17 \mathrm{~m}^{2}$, zero mean assumed. Applying expression (iv) $\sigma^{2}=8.56 \mathrm{~m}^{2}, A=108 \mathrm{~m}^{2}$ and $N=1409$.

Where $\sigma_{s}^{2}$ is assumed to be zero (Kerster and Levin, 1968; Richards and Ibrahim, 1978) expression (ii) is equivalent to expression (iv); otherwise expression (ii) leads to underestimates (fig. 2). Levin and Kerster (1969a) estimated neighbourhood sizes for four colonies of Liatris aspersa. They found that pollen dispersal variance was inversely related to plant density (table 1). Axial $\sigma_{s}^{2}=2.43 \mathrm{~m}^{2}$ was estimated by the cheesecloth method and expression (ii) was employed to give the neighbourhood areas and sizes shown in table 1 . Levin and Kerster re-estimated neighbourhood areas, under the assumption that $\sigma_{s}^{2}=0$, by $A=4 \pi \sigma_{p}^{2}$ so that the full densitydependent effect of $\sigma_{p}^{2}$ was apparent. The decrease in area increases with plant density which is unexpected as a density-independent component has been removed. New estimates are given in table 1 using expression (iv) and $A=4 \pi \sigma_{p}^{2} / 2$ when seed dispersal is discounted; neighbourhood areas and

TABLE 1

Data for Liatris aspersa from Levin and Kerster (1969a). (a) as published; $\sigma_{s, \text { axial }}^{2}=2.43$. (b) recalculated: see text for details

\begin{tabular}{|c|c|c|c|c|}
\hline Colony & I & II & III & IV \\
\hline $\begin{array}{l}\text { Density (plants } \mathrm{m}^{-2} \text { ) } \\
\text { Pollinator flights: } \\
\text { number scored }\end{array}$ & 1 & $3 \cdot 25$ & 5 & 11 \\
\hline $\begin{array}{l}n_{p} \\
\sum p^{2} \\
\Sigma p^{2} / n_{p} \\
\sigma_{p, a x i a l}^{2}\end{array}$ & $\begin{array}{r}393 \\
1806 \cdot 90 \\
4 \cdot 60 \\
2 \cdot 30\end{array}$ & $\begin{array}{r}337 \\
837 \cdot 21 \\
2 \cdot 48 \\
1 \cdot 24\end{array}$ & $\begin{array}{r}346 \\
463 \cdot 39 \\
1.34 \\
0.67\end{array}$ & $\begin{array}{r}538 \\
387 \cdot 11 \\
0 \cdot 72 \\
0 \cdot 36\end{array}$ \\
\hline (a) $\left(\sigma^{2}+\sigma^{2}\right)$ & & & & \\
\hline$A=4 \pi\left(\frac{\sigma_{p}^{-}+\sigma_{s}^{s}}{2}\right)$ & 30 & 23 & 19 & 17 \\
\hline$A=4 \pi \sigma_{p}^{2}$ & 29 & 16 & $8 \cdot 4$ & $4 \cdot 5$ \\
\hline Difference & 1 & 7 & $10 \cdot 6$ & $12 \cdot 5$ \\
\hline $\begin{array}{l}N(\text { pollen and seed }) \\
N \text { (pollen only })\end{array}$ & $\begin{array}{l}30 \\
29\end{array}$ & $\begin{array}{l}75 \\
51\end{array}$ & $\begin{array}{l}97 \\
42\end{array}$ & $\begin{array}{r}191 \\
50\end{array}$ \\
\hline $\begin{array}{l}\text { (b) } \\
A=4 \pi\left(\frac{1}{2} \sigma_{p}^{2}+\sigma_{s}^{2}\right) \\
A=4 \pi\left(\frac{1}{2} \sigma_{p}^{2}\right)\end{array}$ & $\begin{array}{l}45 \\
14\end{array}$ & $\begin{array}{r}38 \\
8\end{array}$ & $\begin{array}{c}35 \\
4 \cdot 2 \\
\end{array}$ & $\begin{array}{l}33 \\
2 \cdot 3\end{array}$ \\
\hline Difference & 31 & 30 & $30 \cdot 8$ & $30 \cdot 7$ \\
\hline $\begin{array}{l}N \text { (pollen and seed) } \\
N \text { (pollen only) }\end{array}$ & $\begin{array}{l}45 \\
14\end{array}$ & $\begin{array}{c}123 \cdot 5 \\
28\end{array}$ & $\begin{array}{r}175 \\
21\end{array}$ & $\begin{array}{r}363 \\
25\end{array}$ \\
\hline
\end{tabular}


sizes are altered substantially and the difference in area on removing the density-independent component is constant.

In a later paper (Schaal and Levin, 1978) data are presented for Liatris cylindracea in the same locality. Absolute $\sigma_{p}^{2}=0.96 \mathrm{~m}^{2}$ and axial $\sigma_{s}^{2}=$ $4.8 \mathrm{~m}^{2}$ so that the neighbourhood area is $33 \mathrm{~m}^{2}$ using expression (ii). Application of expression (iv) almost doubles neighbourhood area to $63 \mathrm{~m}^{2}$. A comparison is made with the results for $L$. aspersa which, however, bear little resemblance to those in the 1969 paper: zero mean variances for $L$. cylindracea are compared with raw mean variances for $L$. aspersa and absolute pollen variances are combined with axial seed variances for both species to yield a new neighbourhood area of $24 \mathrm{~m}^{2}$ for $L$. aspersa at a density of 5 plants $\mathrm{m}^{-2}$.

These examples illustrate how the various methods of combining pollen and seed dispersal variances can lead to substantial differences in estimates of neighbourhood area and size. In view of the interesting nature of these estimates it is hoped that future papers on plant neighbourhoods will give precise details of how variances have been obtained, corrected (where necessary) and combined to yield the parent-offspring dispersal variance.

\section{REFERENCES}

CRAWFORD, T. J. 1984. What is a population? In Shorrocks, B. (ed.) Evolutionary Ecology, Blackwell Scientific Publications, Oxford, pp. 135-173.

DOBZHANSKY, TH. AND WRIGHT, s. 1943. Genetics of natural populations. X. Dispersion rates in Drosophila pseudoobscura. Genetics, 28, 304-340.

KERSTER, H. W. 1964. Neighborhood size in the rusty lizard, Sceloporus olivaceus. Evolution, $18,445-457$.

KERSTER, H. W. AND LEVIN, D. A. 1968. Neighborhood size in Lithospermum caroliniense. Genetics, 60, 577-587.

KNUTH, D. E. 1981. The Ant of Computer Programming. Vol. 2, Seminumerical Algorithms, (2nd Ed.), Addison-Wesley, Reading, Mass.

LEVIN, D. A. 1978. Pollinator behaviour and the breeding structure of plant populations. In Richards, A. J. (ed.) The Pollination of Flowers by Insects, Academic Press, London, pp. 133-150.

LEVIN, D. A. 1979. Pollinator foraging behaviour: genetic implications for plants. In Solbrig, O. T., Jain, S., Johnson, G. B. and Raven, P. H. (eds.) Topics in Plant Population Biology, MacMillan, London, pp. 131-153.

LEVIN, D. A. AND KERSTER, H. W. 1968. Local gene dispersal in Phlox. Evolution, 22, 130-139.

LEVIN, D. A. AND KERSTER, H. W. 1969a. Density-dependent gene dispersal in Liatris. Am. Nat., 103, 61-74.

LEVIN, D. A. AND KERSTER, H. W. 1969b. The dependence of bee-mediated pollen and gene dispersal upon plant density. Evolution, 23, 560-571.

LEVIN, D. A. AND KERSTER, H. W. 1971. Neighborhood structure in plants under diverse reproductive methods. Am. Nat., 105, 345-354.

LEVIN, D. A. AND KERSTER, H. W. 1974. Gene flow in seed plants. Evolutionary Biology, 7, 139-220.

LEVIN, D. A. AND KERSTER, H. W. 1975. The effect of gene dispersal on the dynamics and statics of gene substitution in plants. Heredity, 35, 317-336.

MACKAY, I. J. 1980. Population Genetics of Papaver dubium. Unpublished Ph.D. Thesis, University of Birmingham.

RICHARDS, A. J. AND IBRAHIM, H. 1978. Estimation of neighbourhood size in two populations of Primula veris. In Richards, A. J., (ed.) The Pollination of Flowers by Insects, Academic Press, London, pp. 165-174.

ROHLF, F. J. AND SCHNELL, G. D. 1971. An investigation of the isolation-by-distance model. Am. Nat., 105, 295-324.

SCHAAL, B. A. 1980. Measurement of geneflow in Lupinus texensis. Nature, 284, 450-451. 
SCHAAL, B. A. AND LEVIN, D. A. 1978. Morphological differentiation and neighborhood size in Liatris cylindracea. Am. J. Bot., 65, 923-928.

SCHMITT, J. 1980. Pollinator foraging behavior and gene dispersal in Senecio (Compositae). Evolution, 34, 934-943.

WRIGHT, S. 1943. Isolation by distance. Genetics, $28,114-138$.

WRIGHT, S. 1946. Isolation by distance under diverse systems of mating. Genetics, 31, 39-59. WRIGHT, S. 1969. Evolution and the Genetics of Populations. Vol. 2. The Theory of Gene Frequencies. University of Chicago Press, Chicago.

WRIGHT, s. 1978. Evolution and the Genetics of Populations. Vol. 4, Variability within and among Natural Populations. University of Chicago Press, Chicago. 